\title{
A positive feedback loop of p53/miR-19/TP53INP1 modulates pancreatic cancer cell proliferation and apoptosis
}

\author{
XIAOFANG WANG, LEI WANG, QINGJIANG MO, ANKUI JIA, YUQIAN DONG and GUOQIANG WANG \\ Department of Clinical Laboratory, The First Affiliated Hospital of Xinxiang \\ Medical University, Weihui, Henan 453100, P.R. China
}

Received May 20, 2015; Accepted June 26, 2015

DOI: $10.3892 /$ or.2015.4361

\begin{abstract}
Pancreatic cancer is a common malignancy whose prognosis and treatment of pancreatic cancer is extremely poor, with only $20 \%$ of patients reaching two years of survival. Previous findings have shown that the tumor suppressor p53 is involved in the development of various types of cancer, including pancreatic cancer. Additionally, p53 is able to activate TP53INP1 transcription by regulating several phenotypes of cancer cells. Using gain and loss-of-function assays, the aim of the present study was to examine the relationships between $\mathrm{miR}-19 \mathrm{a} / \mathrm{b}$ and cancer development as well as potential underlying mechanisms. The results showed that miR-19a/b identified a positive feedback regulation of p53/TP53INP1 axis. Additionally, p53 upregulated the TP53INP1 level in pancreatic cancer cells. However, overexpressed miR-19a/b partially restored the TP53 function in the pancreatic cancer cells while miR-19a/b downregulated TP53INP1 protein by directly targeting 3'UTR of its mRNA at the post-transcriptional level. In addition, the patient tissues identified that the $\mathrm{miR}-19 \mathrm{a} / \mathrm{b}$ level in pancreatic cancer tissues was conversely correlated with TP53 and TP53INP1 expression. The results provide evidence for revealing the molecular mechanism involved in the development of pancreatic cancer and may be useful in the identification of new therapeutic targets for pancreatic cancer.
\end{abstract}

\section{Introduction}

Pancreatic cancer (PC) is one of the most common malignancies (1). More than 230,000 cases are diagnosed worldwide annually, mainly in developed countries and with a slight male predominance (2). As a result, the prognosis of pancreatic cancer is extremely poor: the 5-year survival rate is $6 \%$, with only $20 \%$ of patients reaching two years of survival (3). A

Correspondence to: Professor Xiaofang Wang, Department of Clinical Laboratory, The First Affiliated Hospital of Xinxiang Medical University, No. 88 Health Road, Weihui, Henan 453100, P.R. China

E-mail: wangxf0413@126.com

Key words: pancreatic cancer, miR-19a/b, TP53, TP53INP1 number of risk factors such as smoking, diabetes and chronic pancreatitis have been identified in case-control and cohort studies, whereas at present results remain inconclusive, mainly due to the role of candidate genes (4).

The TP53 protein is a key tumor suppressor (5) that responds to various cell stresses, such as DNA damage, hypoxia, metabolic stress and oncogene activation (6). Previous findings have shown that the tumor suppressor TP53 is involved in the cancer development of various cancer types including breast, colon and pancreatic cancer, while it is frequently mutated in pancreatic cancer, and these mutations result in the absence or dysfunction of the p53 protein (7). TP53 also interacts with numerous cell proteins in the control of programmed cell death, including tumor protein $\mathrm{p} 53$-induced nuclear protein 1 (TP53INP1) transcription, which regulates several phenotypes of cancer cells involved in pancreatic cancer. TP53INP1 is a key stress-response protein that is highly expressed during pancreatitis (8). Previous findings demonstrated that TP53INP1 deficiency accelerates the progression of pancreatic cancer (9-11). However, the downregulation of TP53INP1 expression has yet to be adequately investigated for pancreatic cancer susceptibility.

MicroRNAs (miRNAs) are small non-coding RNAs that regulate target genes post-transcriptionally through complementary binding to their target mRNAs to induce translational silencing or degradation (12). miRNAs have emerged as oncogenes or tumor suppressors in networks that establish regulatory circuits (13), which are involved in many key cell processes, such as cell growth, proliferation and death. The miR-17-92 cluster first attracted attention following a series of observations that linked it with cancer pathogenesis. It was also shown to be overexpressed in many types of cancer, including lung, colon and breast cancer and neuroblastoma, and pancreatic cancer (14). miR-17-92, reduced by p53 at the transcriptional level, has an important function in cell apoptosis for the regulation of cancer development (15). miR-19a and miR-19b (miR-19a/b) are located in the miR-17-92 cluster because they were recently identified to be the most important miRNAs in this cluster (16). Thus, we aimed to examine the relationships between miR-19a/b and cancer development and the potential mechanisms. Using gain and loss-of-function assays, we found that $\mathrm{miR}-\mathrm{a} / \mathrm{b}$ function was downregulated by TP53 expression. Furthermore, the tumor suppressor TP53INP1 was demonstrated to be a direct target of miR-19a/b. Our findings provide 
mechanistic insight into the function of $\mathrm{miR}-19 \mathrm{a} / \mathrm{b}$ and provide evidence regarding the molecular mechanism involved in the development of pancreatic cancer, which may be useful in the identification of new therapeutic targets for pancreatic cancer.

\section{Materials and methods}

Clinical human pancreatic cancer specimens and cell lines. Sixteen paired human pancreatic cancer and adjacent pancreatic tissue were confirmed and examined to detect miR-19a/b, TP53 and TP53INP1 expression levels. RNA was isolated from the tissue samples according to the manufacturer's instructions.

The human PANC-1 and PC-3 pancreatic cancer cell lines were obtained from the American Type Culture Collection (ATCC; Manassas, VA, USA). The two cell lines were cultured in RPMI-1640 (Sigma, St. Louis, MO, USA), supplemented with $10 \%(\mathrm{v} / \mathrm{v})$ fetal bovine serum, $1 \%$ PS (100 U/ml penicillin and $100 \mu \mathrm{g} / \mathrm{ml}$ streptomycin). The cell lines were maintained in a humidified atmosphere at $37^{\circ} \mathrm{C}$ with $5 \% \mathrm{CO}_{2}$. Transient transfection was performed using the Lipofectamine ${ }^{\mathrm{TM}} 2000$ transfection reagent (Invitrogen Life Technologies, Carlsbad, CA, USA) according to the manufacturer's instructions.

Antibodies, reagents and DNA constructs. Mouse monoclonal antibody against Flag-tag and rabbit polyclonal antibodies against TP53 and TP53INP1 were purchased from Sigma. ASO-miR-19a/b and miR-19a/b were obtained from Applied Biosystems (Carlsbad, CA, USA).

pcMV6/TP53 was constructed by using standard techniques. pcMV6 was digested with $\mathrm{XhoI}$ and KpnI. Two strands were annealed to clone a fragment of the TP53 protein with XhoI and KpnI sites. This construct was inserted into the pcMV6 vector. The two strands were then annealed to clone a fragment of the TP53INP1 3'UTR containing the target site of miR-19a/b with BamHI and XhoI sites. This construct was inserted into a BamHI-XhoI digested EGFP luciferase reporter vector. To generate a mutant containing a mutation in the miR19a/b target site, two strands were annealed and inserted into a BamHI-XhoI digested TP53INP1-3'UTR-mut reporter vector.

In silico analysis was performed using TargetScan software (http://www.targetscan.org/) to determine whether the 3'UTR of human TP53INP1 contained conserved putative target site for miR-19a/b miRNAs.

Luciferase reporter assay. The pancreatic PANC-1 cancer cell lines were co-transfected with ASO-miR-19a/b or miR-19a/b and the wild-type or mutant 3'UTR of TP53INP1 compared with the control class in 48 -well plates. At $48 \mathrm{~h}$ after transfection, the Luciferase EGFP intensity was measured with an F-4500 fluorescence spectrophotometer (Hitachi, Chestertown, MD, USA), and EGFP luciferase activity was normalized to that of RFP.

Reverse transcription-qPCR analysis. Total RNA was extracted by using High Pure RNA Isolation kit (Roche) according to the protocol described by the manufacturer. Reverse transcription-qPCR (RT-qPCR) was carried out using the M-MLV reverse transcriptase (Invitrogen Life Technologies). For the gene and miRNA analysis, PCR was performed at a temperature of $58^{\circ} \mathrm{C}$ for 30 cycles in reaction mixture of $25 \mu \mathrm{l}$ individually of each sample in an iQ5 realtime PCR system (Bio-Rad Laboratories, Hercules, CA, USA). GAPDH served as the control gene, and the human U6 RNA as a control small RNA.

Western blot analysis. Cell lysates were prepared using lysis buffer containing $10 \mathrm{mM}$ Tris- $\mathrm{HCl}$ (pH 7.4), 1\% SDS, $1 \mathrm{mM}$ $\mathrm{Na}_{3} \mathrm{VO}_{4}, 10 \mathrm{mM} \mathrm{NaF}$ and protease inhibitor cocktail (Roche, Mannheim, Germany). Protein expression was analyzed by western blot analysis. The separated proteins by SDS-PAGE (8-15\% gel) were transferred to PVDF membranes followed by immunostaining with the primary antibodies at $4^{\circ} \mathrm{C}$ overnight. Horseradish peroxidase-conjugated goat anti-mouse secondary antibody was then applied at room temperature $\left(25^{\circ} \mathrm{C}\right)$ for $2 \mathrm{~h}$, and the specific protein bands were detected using ECL (Amersham, Piscataway, NJ, USA). After detection of the protein bands, the blot was re-probed with anti-GAPDH antibody to confirm equal loading of the samples. The following antibodies used were TP53 and TP53INP1 at a dilution of 1:3,000 and 1:5,000, respectively.

Cell viability and TUNEL assay. The cell viability and proliferation of PANC-1 and PC-3 cell lines with Si-TP53 or miR-19a/b mimics were determined by 3-(4,5-dimethylthiazolyl-2-yl)-2-5 diphenyl tetrazolium bromide (MTT; Sigma) assay. The cells plated in 96 -well plates at $5 \times 10^{3} /$ well were performed according to the manufacturer's instructions. The cell viability was normalized to that of cells cultured in the culture medium with Si-NC. Three independent experiments (three replicates in each) were performed.

A TdT-mediated dUTP-biotin nick end-labeling (TUNEL) assay was performed in PANC-1 cells by using Roche ${ }^{\circledR}$ TUNEL kit as per the manufacturer's instructions. TUNEL-positive nuclei were calculated at a magnification of x20 under an Olympus IX73 microscope (Tokyo, Japan). The assays were performed three times.

Statistical analysis. The data are presented as the mean \pm standard deviation (SD). Statistical significances were calculated using the Student's t-test. $\mathrm{P} \leq 0.05$ was considered to indicate a statistically significant result.

\section{Results}

TP53 regulates miR-19a/b and TP53INP1 expression in pancreatic cancer cells. TP53 is a tumor-suppressor gene and has a fundamental role in pancreatic cancer cell apoptosis (17). We constructed the pcMV6/TP53 vector or obtained SiTP53 and infected them into the pancreatic cancer cell lines. The results verified that the expression of TP53 protein (upper panel) or mRNA (lower panel) was lower in the cell lines transfected with Si-TP53, compared with Si-NC (Fig. 1A). In addition, TP53 expression was higher in the cell lines transfected with pcMV6/TP53, compared to pcMV6 (Fig. 1A).

To determine whether TP53 proteins are involved in the regulation of miR-19 cluster expression in pancreatic carcinoma, RT-qPCR was employed to measure the $\mathrm{miR}-19 \mathrm{a} / \mathrm{b}$ and TP53INP1 level in PANC-1 cell lines, transfected with SiTP53 or pcMV6/TP53 compared with $\mathrm{Si}-\mathrm{NC}$ or pcMV6, respec- 

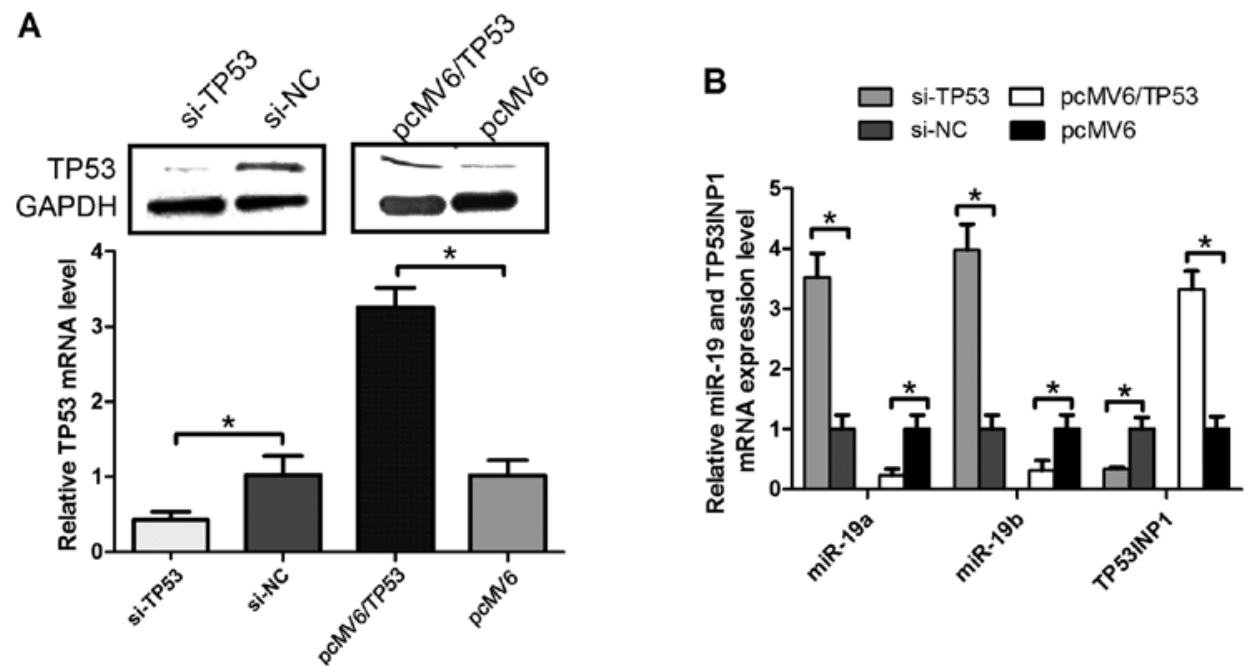

Figure 1. TP53 regulates the endogenous expression of miR-19a/b and TP53INP1. (A) Relative TP53 protein (upper panel) and mRNA (lower panel) levels were expressed in pancreatic cancer cell lines, transfected with Si-TP53 or pcMV6/TP53, compared with Si-NC or pcMV6, respectively. GAPDH served as an internal control. (B) RT-qPCR was employed to measure the miR-19 cluster or TP53INP1 level in the pancreatic cancer cells. "P<0.05.
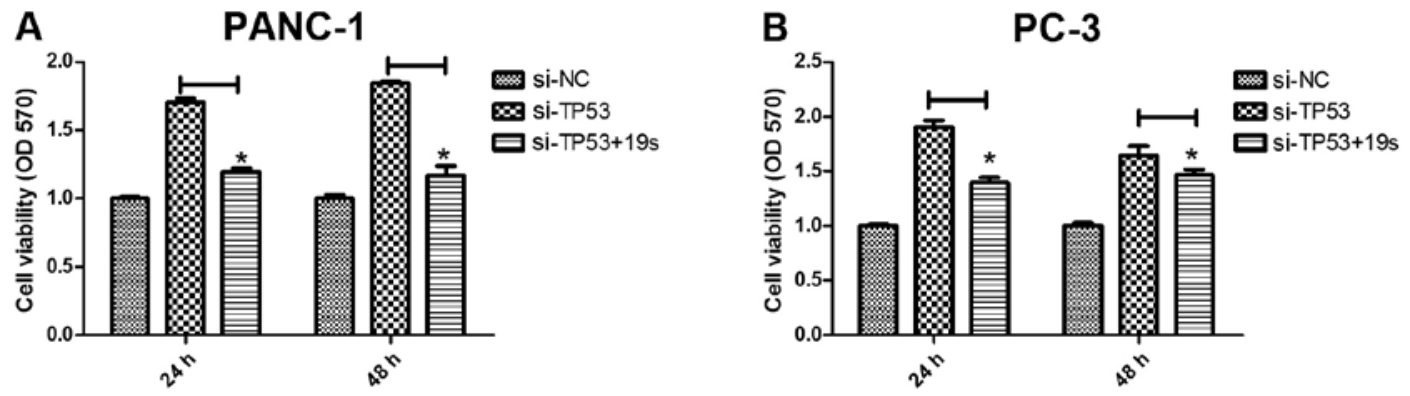

Figure 2. miR-19a/b partly rescues the effects of TP53 protein in pancreatic cancer cells. (A) Cell proliferation was measured in PANC-1 cells at 24 and $48 \mathrm{~h}$ after transfection by the MTT assay. (B) Cell proliferation was measured using Si-NC-, Si-TP53 or miR-19s-infected PC-3 pancreatic cancer cells. *P<0.05.

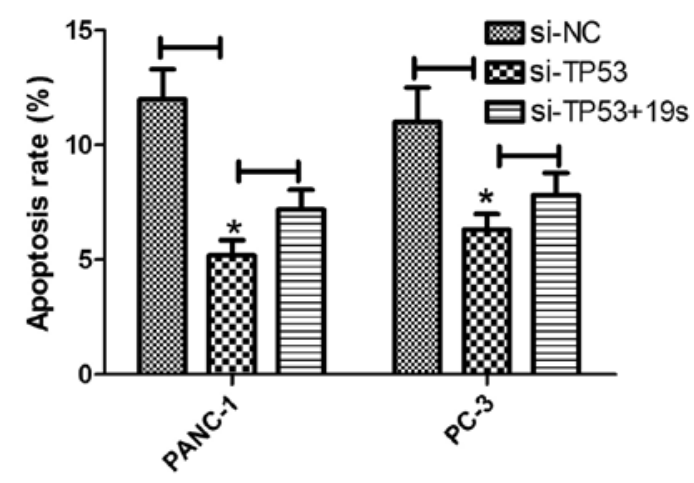

Figure 3. TP53-induced cell apoptosis is partly rescued by miR-19a/b. Cell apoptosis was measured using Si-NC-, Si-TP53, or miR-19s-infected PANC-1 or PC-3 pancreatic cancer cells by TUNEL assay, respectively. Each experiment was independently repeated at least three times. Error bars correspond to the mean $\pm \mathrm{SD} ;{ }^{*} \mathrm{P}<0.05$.

tively. Notably, the expression of TP53 in the pancreatic cancer cell lines did not only conversely correlate with the miR-19a/b level, but also correlated with TP53INP1 mRNA $(\mathrm{P}<0.05)$. The association between TP53 and miR-19a/b or TP53INP1 identified that TP53 regulates miR-19a/b and TP53INP1 expression in pancreatic cancer cells.
Effects of TP53 on cell proliferation in pancreatic cancer are partially restored by $\mathrm{miR}-19 \mathrm{a} / \mathrm{b}$. miR-19a/b was previously regarded as an oncogene and detected to regulate pancreatic development (16). Thus, we hypothesized that the overexpression of miR-19a/b may partially restore TP53 function in pancreatic cancer cell proliferation. To assess the cell viability of miR-19a/b, we transfected Si-NC, Si-TP53, and miR-19s into the PANC-1 and PC-3 pancreatic cancer cell lines and detected absorbances at $570 \mathrm{~nm}$ using a microplate reader. As shown in Fig. 2A, Si-TP53 transfection in the PANC-1 pancreatic cancer cells induced cell proliferation, compared to the Si-NC group $(\mathrm{P}<0.05)$. However, miR-19a/b expression partially restored TP53 function in Si-TP53-transfected cells compared to the Si-TP53 group $(\mathrm{P}<0.05)$ (Fig. 2A). Similar results were observed in PC-3as pancreatic cancer cells (Fig. 2B). These results suggested that miR-19a/b partly restored the role of TP53 protein in pancreatic cancer cell proliferation.

TP53-induced cell apoptosis is partly restored by miR-19a/b. To analyze the miR-19a/b function in pancreatic cancer cell development, Si-NC-, Si-TP53, and miR-19s were transfected into PANC-1 and PC-3 cell lines and a TUNEL assay was employed to detect the role of miR-19a/b in pancreatic cancer. The number of TUNEL-positive cells in PANC-1 
A
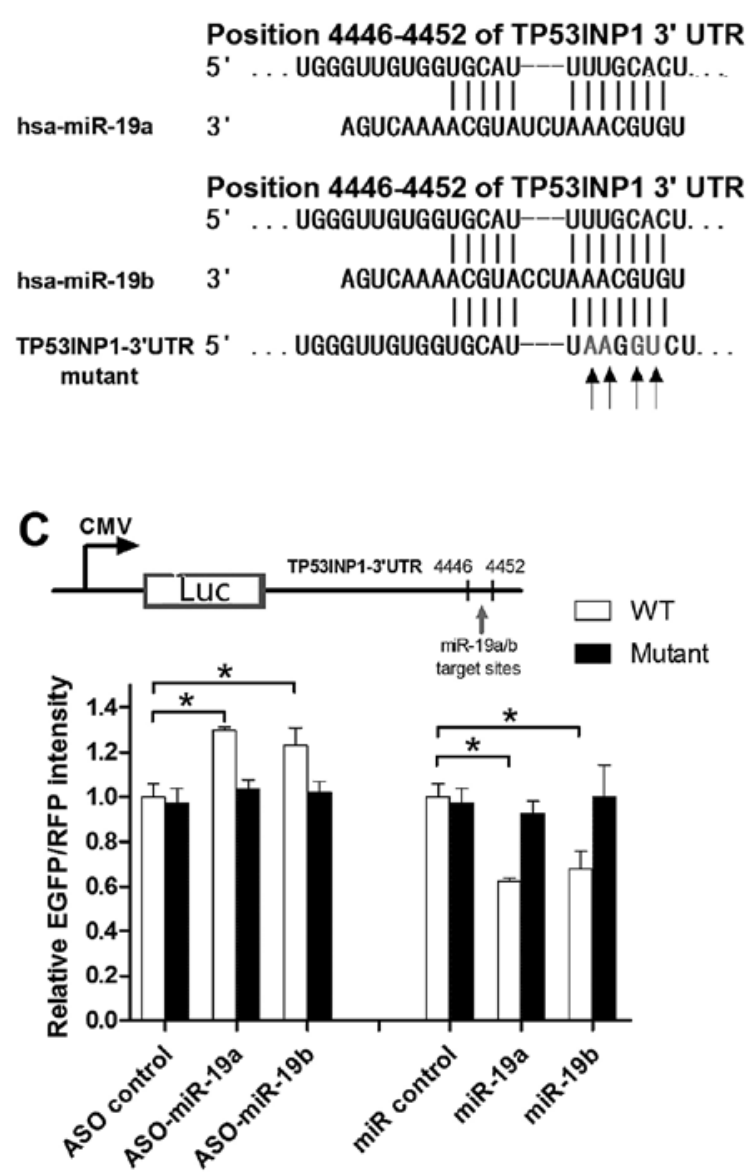

B

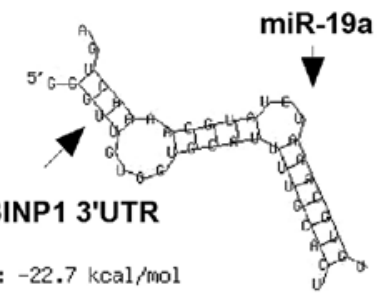

mfe: $-22.7 \mathrm{kcal} / \mathrm{mol}$

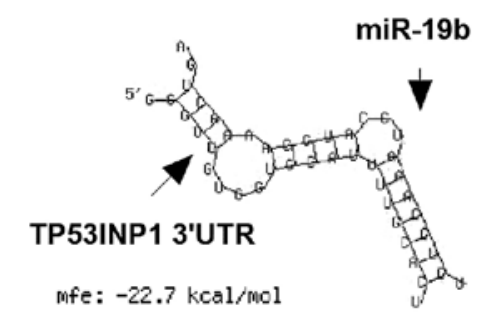

D

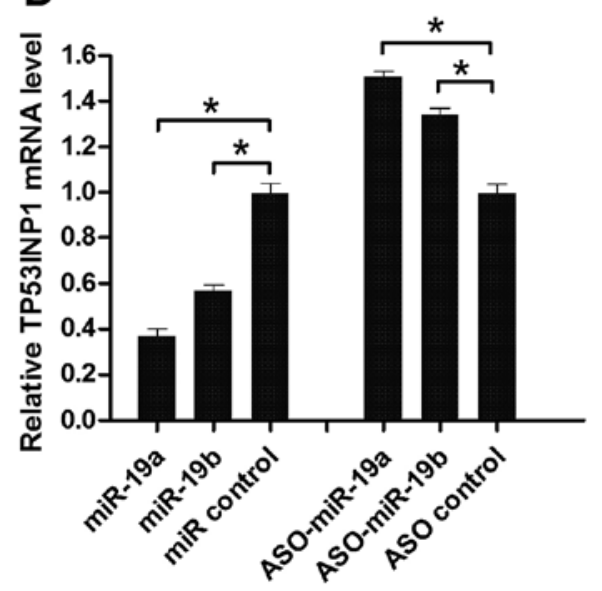

Figure 4. TP53NP1 mRNA is the target of miR-19a/b. (A) The target sites for miR-19a/b in the 3'UTR of TP53NP1 mRNA, and the mutant target sites. (B) The schemes showed the structures between TP53NP1 3'UTR and miR-19a/b. (C) Relative EGFP intensity in pancreatic cancer cells transfected with ASO-miR-19a/b or miR-19a/b compared to their control, was normalized to RFP. (D) RT-qPCR of TP53INP1 level in PANC-1 cell lines infected with the ASO-miR-19a/b, miR-19a/b or negative control. ${ }^{*} \mathrm{P}<0.05$.

transfected with Si-TP53 were significantly elevated in the Si-NC-transfected groups. However, miR-19a/b expression partially restored TP53 function in Si-TP53-transfected cells compared to the Si-TP53 group (Fig. 3, left panel) $(\mathrm{P}<0.05)$. Similar results were observed in pancreatic cancer cells PC-3 as indicated in Fig. 3, right panel.

miR-19a/b directly targets TP53INP1. In silico analysis using TargetScan software (http://www.targetscan.org/) showed that the 3'UTR of human TP53INP1 contains a conserved putative target site for miR-19a/b miRNAs (Fig. 4A). In the present study, miRNA identification and analysis were developed through a primary tool, RNAhybrid (18), in order to identify the available targets according to the secondary structure feature and minimum free energy (MFE) between the miRNAs and target gene. To validate these target sites, the 3'UTR of human TP53INP1 (Homo sapiens, 4446-4452 bp) was amplified and inserted in both orientations downstream of the EGFP luciferase gene in the pcDNA3-control vector, generating sense (S) and antisense (AS) constructs, collectively referred to as pcDNA3 (Fig. 4C, upper panel). One mutant clone (mutant) was prepared in a similar manner. We also obtained the ASO-miR-19a/b to inhibit the endogenous level in the pancreatic cancer cell lines. The transfection of PANC-1 cells with ASO-miR-19a or ASO-miR-19b significantly increased the expression of pcDNA3/EGFP-TP53INP1 compared to the mutant, while with miR-19a or miR-19b a decreased expression of pcDNA3/EGFPTP53INP1 was observed (Fig. 4C, lower panel). Furthermore, miR-19a and miR-19b targeted TP53INP1 mRNA (Fig. 4D).

miR-19a/b represses TP53INP1 level in PC cells. To determine the downregulation effects of $\mathrm{miR}-19 \mathrm{a} / \mathrm{b}$ at the TP53 protein level, we performed western blot assays using an anti-TP53IP1 antibody. As expected, the transient transfection of the PANC-1 pancreatic cancer cell with miR-19a or miR-19b decreased TP53INP1 levels, compared to the miR control (Fig. 5A). However, cells transiently transfected with ASO-miR-19a or ASO-miR-19b showed increased TP53INP1 levels (Fig. 5B). These results suggested that $\mathrm{miR}-19 \mathrm{a} / \mathrm{b}$ miRNAs regulate MXD1 expression in vivo at the post-transcriptional level.

Different expression of miR-19a/b in pancreatic cancer tissues is conversely correlated with the TP53 and TP53INP1 protein. To examine the relationship between miR-19a/b and TP53INP1, 16 paired human pancreatic cancer tissues and adjacent pancreatic tissues were confirmed by pathological analysis and the 


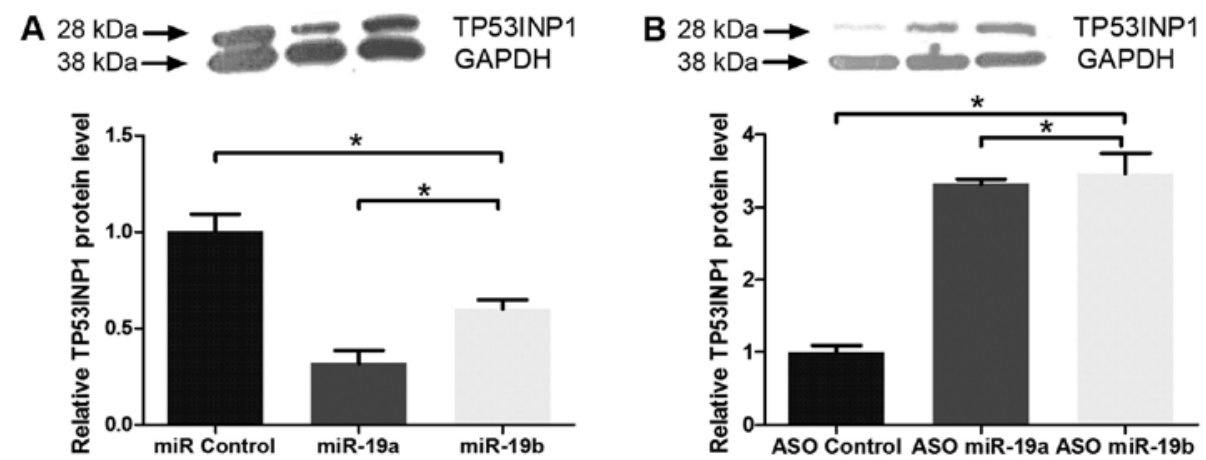

Figure 5. miR-19a/b regulates the TP53INP1 protein in vivo. (A) Representative immunoblot images (upper panel) and quantification (lower panel) of TP53INP1 protein in pancreatic cancer cells transfected with miR-19a/b or miR control. GAPDH served as an internal control. (B) Western blot analysis of TP53INP1 level in pancreatic cancer cells infected with the ASO-miR-19a/b, or ASO control. "P<0.05.
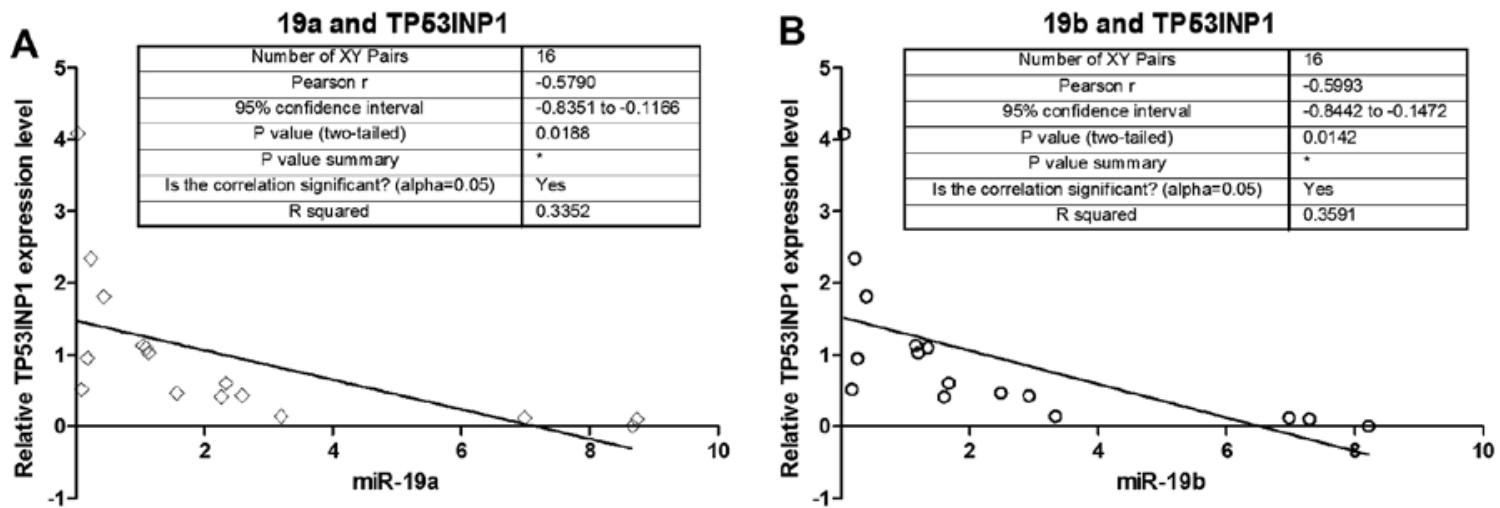

TP53 and 19a

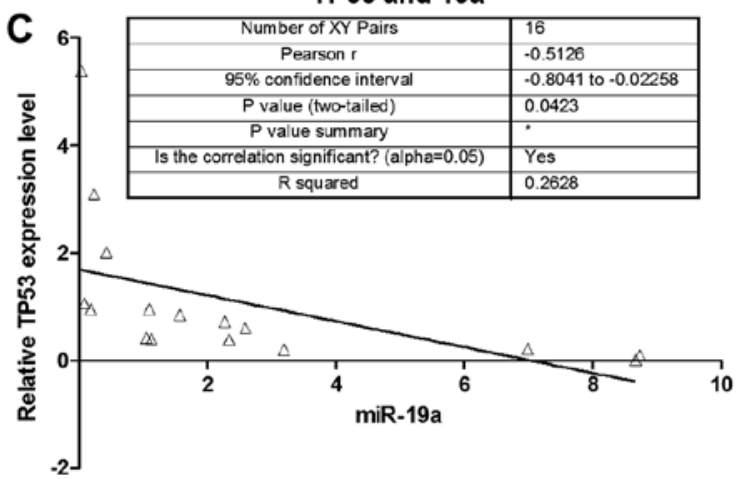

TP53 and 19b

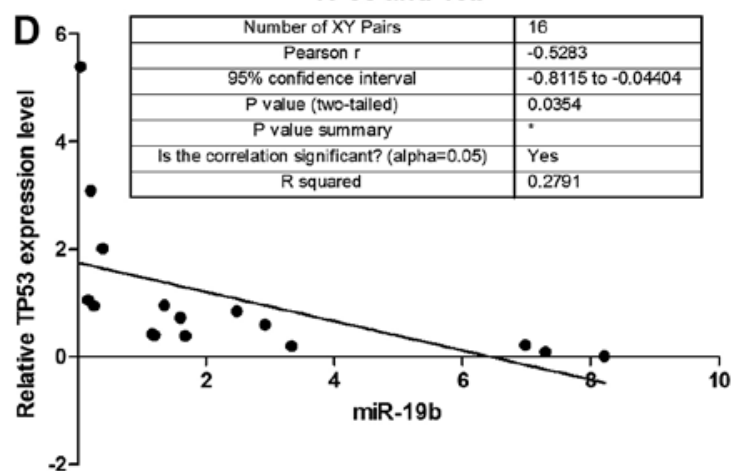

Figure 6. miR-19a/b level in pancreatic cancer tissues is conversely correlates with TP53 and TP53INP1 expression. (A and B) Relative TP53INP1, (A) miR-19a and (B) -19b level in pancreatic cancer tissues was measured by RT-qPCR. (C and D) RT-qPCR was employed to measure the TP53, (C) miR-19a and (D) $-19 b$ level. ${ }^{*} \mathrm{P}<0.05$.

endogenous miR-19a/b and TP53INP1 level was measured using RT-qPCR assay. As shown in Fig. 5A and B, in human tissues, the expression levels of miR-19a and miR-19b were conversely correlated with the TP53INP1 protein. To determine whether miR-19a and miR-19b levels were regulated by TP53, we performed RT-qPCR on 16 paired human pancreatic cancer tissues and adjacent pancreatic tissues. miR-19a and miR-19b expression levels were conversely correlated with the TP53 protein. These results demonstrated that although miR-19a/b is capable of downregulating TP53INP1 expression by binding its $3^{\prime} \mathrm{UTR}$, the overexpression of TP53 is also able to reduce miR19a/b expression levels, indicating a potential miRNA target gene regulatory loop between miR-19a/b TP53, and TP53INP1.

\section{Discussion}

Although miR-19a/b has been reported to have a broad and significant roles in many types of cancer cells $(16,19,20)$, the exact function and the potential mechanisms in pancreatic cancer remain to be investigated. The present study identified the direct targets and the underlying functions of miR-19a/b by using bioinformatics tools and gene manipulations using pancreatic cancer cell. In summary, our novel findings are: i) overexpression of miR-19a/b partly rescued TP53-induced cell apoptosis to promote cell proliferation; ii) the luciferase reporter assays, RT-qPCR and western blot analysis showed that miR-19a/b directly downregulated TP53INP1 at the 
mRNA and protein levels; iii) and thus, the miR-19a/b family exerts important functions in pancreatic cancer development by regulating endogenous TP53INP3 protein and is regulated by TP53 expression in vivo.

The role of the tumor-suppressor gene, TP53 has been investigated in considerable depth in previous studies (21-23). In the present study, we observed that miR-19a/b expression was regulated by endogenous TP53 protein in most of the randomly selected pancreatic cancer samples and the overexpression of miR-19a and miR-19b partly rescued the TP53-mediated suppression of the development of pancreatic cancer. Furthermore, MTT assays were performed to examine the relationships between miR-19a/b miRNAs and cell viability in pancreatic cancer using transient miR-19a/b-expressing cells. Moreover, an anti-apoptotic function was observed in the present study using TUNEL assays, which was consistent with previous studies showing that $\mathrm{miR}-19 \mathrm{a} / \mathrm{b}$ acts as an oncomiR in pancreatic cancers.

TP53INP1 is a key tumor suppressor that modulates p53 to induce tumor cell death (24-26). We showed in a present study that miR-19a/b directly targeted TP53INP1 3'UTR and regulated the TP53INP1 mRNA and protein level in pancreatic cancer cells. Moreover, pancreatic cancer cells transiently transfected with si-TP53 triggered miR-19s expression, and that reduced endogenous TP53INP1 gene expression enhanced cell viability to inhibit cell apoptosis. However, previous findings have shown that TP53 directly binds to the TP53INP1 promoter region and triggers TP53INP1 expression in various cancer types $(27,28)$. The patient tissues showed that miR-19a/b level in pancreatic cancer tissues was conversely correlated with the expression of TP53 and TP53INP1. The results of the present study suggested that TP53INP1 regulated by miR-19s is one of the molecular mechanisms driving p53 to induce cell apoptosis.

In conclusion, the results suggest that overexpression of the miR-19a/b family partially restored cell apoptosis induced by the endogenous TP53 protein. Therefore, miR-19a/b improved cell proliferation through the repression of novel target gene TP53INP1. The present findings indicate that the positive regulatory network between TP53, miR-19a/b and TP53INP1 may be important in inhibiting the expression levels of oncogenic miRNAs and may provide new insights into the mechanism involved in the development of pancreatic cancer.

\section{References}

1. Siegel RL, Miller KD and Jemal A: Cancer statistics, 2015. CA Cancer J Clin 65: 5-29, 2015.

2. Tempero MA, Arnoletti JP, Behrman SW, Ben-Josef E, Benson AB III, Casper ES, Cohen SJ, Czito B, Ellenhorn JD, Hawkins WG, et al; National Comprehensive Cancer Networks: Pancreatic Adenocarcinoma, version 2.2012: Featured updates to the NCCN Guidelines. J Natl Compr Canc Netw 10: 703-713, 2012 .

3. Hidalgo M: Pancreatic cancer. N Engl J Med 362: 1605-1617, 2010.

4. Burki TK: Whole-genome analysis of pancreatic cancer. Lancet Oncol 16: e161, 2015.

5. Levine AJ, Finlay CA and Hinds PW: P53 is a tumor suppressor gene. Cell 116: S67-S69, 61 p following S69, 2004.

6. Whibley C, Pharoah PD and Hollstein M: p53 polymorphisms: Cancer implications. Nat Rev Cancer 9: 95-107, 2009.

7. Petitjean A, Achatz MI, Borresen-Dale AL, Hainaut $P$ and Olivier M: TP53 mutations in human cancers: Functional selection and impact on cancer prognosis and outcomes. Oncogene 26: 2157-2165, 2007.
8. Tomasini R, Samir AA, Vaccaro MI, Pebusque MJ, Dagorn JC, Iovanna JL and Dusetti NJ: Molecular and functional characterization of the stress-induced protein (SIP) gene and its two transcripts generated by alternative splicing. SIP induced by stress and promotes cell death. J Biol Chem 276: 44185-44192, 2001.

9. N'guessan P, Pouyet L, Gosset G, Hamlaoui S, Seillier M, Cano CE, Seux M, Stocker P, Culcasi M, Iovanna JL, et al: Absence of tumor suppressor tumor protein 53-induced nuclear protein 1 (TP53INP1) sensitizes mouse thymocytes and embryonic fibroblasts to redox-driven apoptosis. Antioxid Redox Signal 15: 1639-1653, 2011.

10. Cano CE, Gommeaux J, Pietri S, Culcasi M, Garcia S, Seux M, Barelier S, Vasseur S, Spoto RP, Pébusque MJ, et al: Tumor protein 53-induced nuclear protein 1 is a major mediator of p53 antioxidant function. Cancer Res 69: 219-226, 2009.

11. Gommeaux J, Cano C, Garcia S, Gironella M, Pietri S, Culcasi M, Pébusque MJ, Malissen B, Dusetti N, Iovanna J, et al: Colitis and colitis-associated cancer are exacerbated in mice deficient for tumor protein 53-induced nuclear protein 1. Mol Cell Biol 27: 2215-2228, 2007.

12. Bartel DP: MicroRNAs: Target recognition and regulatory functions. Cell 136: 215-233, 2009.

13. Croce CM: Causes and consequences of microRNA dysregulation in cancer. Nat Rev Genet 10: 704-714, 2009.

14. Morimura R, Komatsu S, Ichikawa D, Takeshita H, Tsujiura M, Nagata H, Konishi H, Shiozaki A, Ikoma H, Okamoto K, et al: Novel diagnostic value of circulating miR-18a in plasma of patients with pancreatic cancer. Br J Cancer 105: 1733-1740, 2011.

15. Yan HL, Xue G, Mei Q, Wang YZ, Ding FX, Liu MF, Lu MH, Tang Y, Yu HY and Sun SH: Repression of the miR-17-92 cluster by 553 has an important function in hypoxia-induced apoptosis. EMBO J 28: 2719-2732, 2009.

16. Wu Q, Yang Z, An Y, Hu H, Yin J, Zhang P, Nie Y, Wu K, Shi Y and Fan D: MiR-19a/b modulate the metastasis of gastric cancer cells by targeting the tumour suppressor MXD1. Cell Death Dis 5: e1144, 2014.

17. Naccarati A, Pardini B, Polakova V, Smerhovsky Z, Vodickova L, Soucek P, Vrana D, Holcatova I, Ryska M and Vodicka P: Genotype and haplotype analysis of TP53 gene and the risk of pancreatic cancer: An association study in the Czech Republic. Carcinogenesis 31: 666-670, 2010.

18. Krüger $\mathbf{J}$ and Rehmsmeier M: RNAhybrid: microRNA target prediction easy, fast and flexible. Nucleic Acids Res 34 (Web Server): W451-W454, 2006

19. Kurokawa K, Tanahashi T, Iima T, Yamamoto Y, Akaike Y, Nishida K, Masuda K, Kuwano Y, Murakami Y, Fukushima M, et al: Role of miR-19b and its target mRNAs in 5-fluorouracil resistance in colon cancer cells. J Gastroenterol 47: 883-895, 2012.

20. Zhang J, Xiao Z, Lai D, Sun J, He C, Chu Z, Ye H, Chen S and Wang J: miR-21, miR-17 and miR-19a induced by phosphatase of regenerating liver-3 promote the proliferation and metastasis of colon cancer. Br J Cancer 107: 352-359, 2012.

21. Harris CC and Hollstein M: Clinical implications of the p53 tumor-suppressor gene. N Engl J Med 329: 1318-1327, 1993.

22. Green DR and Kroemer G: Cytoplasmic functions of the tumour suppressor p53. Nature 458: 1127-1130, 2009.

23. Venkatanarayan A, Raulji P, Norton W, Chakravarti D, Coarfa C, Su X, Sandur SK, Ramirez MS, Lee J, Kingsley CV, et al: IAPP-driven metabolic reprogramming induces regression of p53-deficient tumours in vivo. Nature 517: 626-630, 2015.

24. Seillier M, Peuget S, Gayet O, Gauthier C, N'Guessan P, Monte M, Carrier A, Iovanna JL and Dusetti NJ: TP53INP1, a tumor suppressor, interacts with LC3 and ATG8-family proteins through the LC3-interacting region (LIR) and promotes autophagydependent cell death. Cell Death Differ 19: 1525-1535, 2012.

25. Peuget S, Bonacci T, Soubeyran P, Iovanna J and Dusetti NJ: Oxidative stress-induced 53 activity is enhanced by a redoxsensitive TP53INP1 SUMOylation. Cell Death Differ 21: 1107-1118, 2014

26. Tomasini R, Seux M, Nowak J, Bontemps C, Carrier A, Dagorn JC, Pébusque MJ, Iovanna JL and Dusetti NJ: TP53INP1 is a novel p73 target gene that induces cell cycle arrest and cell death by modulating p73 transcriptional activity. Oncogene 24: 8093-8104, 2005

27. Okamura S, Arakawa H, Tanaka T, Nakanishi H, Ng CC, Taya Y, Monden M and Nakamura Y: p53DINP1, a p53-inducible gene, regulates p53-dependent apoptosis. Mol Cell 8: 85-94, 2001.

28. Tomasini R, Samir AA, Pebusque MJ, Calvo EL, Totaro S, Dagorn JC, Dusetti NJ and Iovanna JL: P53-dependent expression of the stress-induced protein (SIP). Eur J Cell Biol 81: 294-301, 2002 . 